Supporting Information

\title{
Tuning Plasmonic Coupling from Capacitive to Conductive Regimes via Atomic Control of Dielectric Spacing
}

\author{
Sharmin Haq, Tefera E. Tesema, Bisweswar Patra, Eric Gomez and \\ Terefe G. Habteyes*
}

Center for High Technology Materials, Department of Chemistry and Chemical Biology, University of New Mexico, Albuquerque, New Mexico 87131, USA

\section{Content}

17 pages that include the following descriptions, 13 figures with captions and references

1. Sample preparations and characterizations

1. Conformal evaporation of gold

2. Atomic layer deposition of aluminum oxide on the gold film

3. Preparation of gold nanorods for single particle spectroscopy

4. Single particle dark-field scattering spectroscopy

2. Finite difference time domain simulations and supporting experimental results 


\section{Sample preparations and characterizations}

\subsection{Conformal evaporation of gold}

Gold film (AuF) of thickness $\sim 50 \mathrm{~nm}$ has been evaporated on Si substrate with $2 \mathrm{~nm} \mathrm{Ti}$ adhesion layer using conformal deposition of e-beam evaporation. In this procedure, the mount is not on top of the crucible, rather the sample is mounted in a holder rotating at an angle from a closer distance with respect to the crucible. Au and Ti are evaporated at rates of 0.3 and $0.5 \AA / s$, respectively, at vacuum pressure of $5 \times 10^{-7}$ Torr. The surface roughness ( $\mathrm{rms}$ ) of the AuF is 1.2 $\mathrm{nm}$ as determined using atomic force microscope (Veeco Quadrex D3100).

\subsection{Atomic layer deposition of aluminum oxide on the gold film}

Aluminum oxide film is deposited on the AuF using the atomic layer deposition (ALD) system of Picosun. Trimethylaluminium (TMA) and $\mathrm{H}_{2} \mathrm{O}$ vapors are pulsed alternately through the reaction chamber utilizing $\mathrm{N}_{2}$ as carrier gas at a flow rate of $150 \mathrm{sccm}$ and $200 \mathrm{sccm}$ for TMA and $\mathrm{H}_{2} \mathrm{O}$, respectively. The pressure inside the reaction chamber is 4 Torr with the growth temperature of $100{ }^{\circ} \mathrm{C}$. The TMA reactant exposure time (pulse time) is $0.5 \mathrm{~s}$, and is followed by $\mathrm{N}_{2}$ purging for $10 \mathrm{~s}$. The $\mathrm{H}_{2} \mathrm{O}$ reactant exposure time is $0.7 \mathrm{~s}$, followed by $\mathrm{N}_{2}$ purging for $20 \mathrm{~s}$. The thickness of the deposited aluminum oxide thickness is measured using variable angle spectroscopic ellipsometry (VASE) [J. A. Woolam Co., Inc.] and the accompanying software. Spectroscopic scans in the range of $600-800 \mathrm{~nm}$ at $10 \mathrm{~nm}$ intervals are performed in the $60^{\circ}-70^{\circ}$ range at $10^{\circ}$ interval relative to the normal to the sample surface. Ellipsometry data are analyzed using a layer model, where the oxide layer thickness is fit using Cody-Lorentz oscillator provided by VASE software.

Transmission electron microscope (TEM) is used for imaging the cross section of a representative sample. An FEI Tecnai F30 S/TEM operated at $300 \mathrm{kV}$ in TEM mode is used with a 4k Ultrascan camera. Before taking the TEM images, thin cross section $(<100 \mathrm{~nm}$ thick $)$ of the sample is prepared by focused ion beam (FIB) using NOVA NANOLAB 600 dual beam. In the $\mathrm{FIB}, \mathrm{Pt}$ is deposited in two phase as a protection layer on top of the $\mathrm{A}_{2} \mathrm{O}_{3}$ coated $\mathrm{Au}$ film. In the first phase, $\mathrm{Pt}$ is deposited by electron beam with low acceleration voltage $(\sim 5 \mathrm{kV})$ to protect the top layer. Electron beam protective coating of carbon rich Pt grows relatively slowly, and conforms to high aspect ratio structures as it is deposited. In the second phase, $\mathrm{Pt}$ is deposited by ion beam at high accelerating voltage $(\sim 30 \mathrm{kV})$.

\subsection{Preparation of gold nanorods (AuNRs) for single particle spectroscopy}

Colloidal solution of AuNRs $(40 \mathrm{~nm} \times 80 \mathrm{~nm})$ with cetyl trimethylammonium bromide (CTAB) was obtained from Nanopartz Inc. After at least ten times dilution of the original solution in ultrapure water, about $50 \mu \mathrm{L}$ was drop-casted on the film that was placed on a shaker. After one minute of shaking, the solution was blown off with $\mathrm{N}_{2}$ gas before it dries completely to avoid particle aggregation during drying. This process results in the distribution of isolated AuNRs on the film with large separation from neighboring nanorods. This is confirmed using atomic force 
microscope (AFM) scan images. For example, in most cases no AuNRs are obtained when $10 \mu \mathrm{m}$ $\times 10 \mu \mathrm{m}$ area is scanned randomly on the sample, and when they are found, there is large separation between the AuNRs as shown in Figure S1.
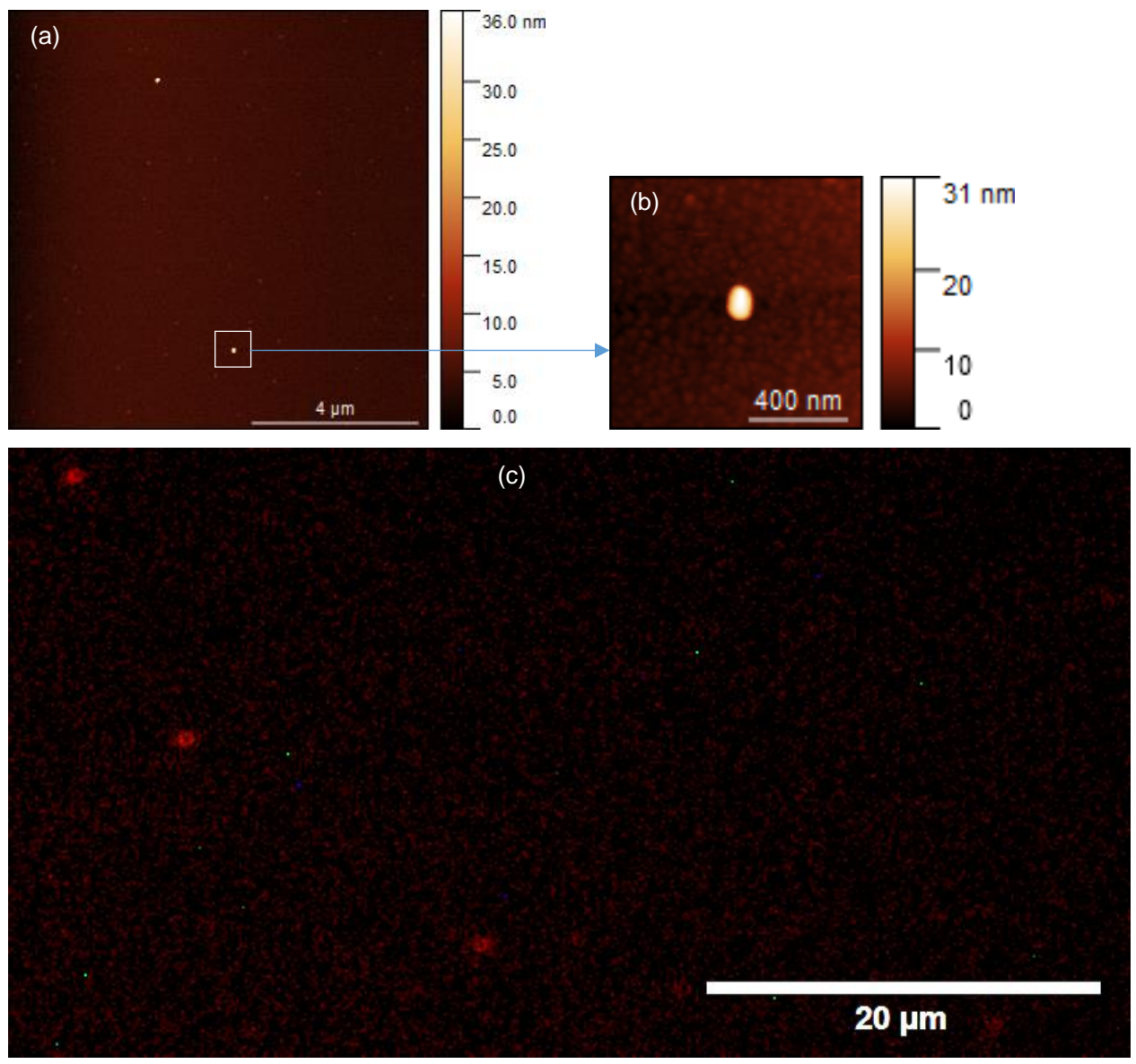

Figure S1 (a) AFM topography of AuNRs on gold film. (b) AFM image showing the AuNR within the square. (c) Dark-field image of AuNRs on gold film for the same sample as in (a) but not necessarily the same location.

\subsection{Single particle dark-field scattering spectroscopy}

In addition to the sample preparation procedures that results in the distribution of isolated AuNRs as described above, the structure and brightness of the dark-field images as well as the intensity and shape of the spectra are used in assigning a spectrum to individual gold nanorods. For example the dark-field images of individual AuNRs deposited directly on gold film have donut shape (see Figure S1) and weaker scattering intensity than aggregates. The single particle darkfield scattering measurement is carried out using the GX51 Olympus microscope. The sample is excited with $100 \mathrm{~W}$ halogen white light source using dark-field microscope objective (MPLFLN100XBD, Olympus) with 0.9 numerical aperture (NA). Dark-field images of the 
individual AuNRs coupled to AuF are obtained by directing $10 \%$ of the signal to the camera (Olympus UC30 or XM10) attached to the microscope. The corresponding scattering spectra are recorded by centering the particle to the focus of the objective, and directing $90 \%$ of the light collected from the sample to the spectrometer (IsoPlane Spectrograph of Princeton Instruments), which uses a thermoelectrically cooled $\left(-75^{\circ} \mathrm{C}\right)$ and back-illuminated, deep depletion CCD camera.

The IsoPlane spectrograph uses $320 \mathrm{~mm}$ focal length and $150 \mathrm{G} / \mathrm{mm}$ ruled grating with 800 $\mathrm{nm}$ blaze wavelength. The back-illuminated, deep depletion CCD camera combined with the blazing wavelength of the grating offer the highest detection quantum efficiency in the nearinfrared regions, peaking at $\sim 800 \mathrm{~nm}$ and extending to the silicon cut off region $(\sim 1.1 \mathrm{eV})$. In our experiment, the peak energies of the plasmon resonances range from $1.25 \mathrm{eV}$ to $2.02 \mathrm{eV}$. Clearly, the detection efficiency is not constant across this spectral range, and hence absolute intensity comparison requires intensity calibration, which is beyond this work.

\section{Finite difference time domain simulations and supporting experimental results}

The scattering cross-section of the AuNRs coupled to gold film is calculated using finite difference time domain (FDTD) method of electromagnetic simulation. The simulation is performed using the commercial software package by Lumerical Solutions, Inc. As mentioned above, the sample is excited by focusing unpolarized white light source using $0.9 \mathrm{NA}$. As a result, different orientation of excitation field with respect to the AuNR axes are considered in the initial simulation using Gaussian beam source with NA specification the same as in the experiment.

The different possible orientations of the AuNR with respect of the excitation field (E) vector at $45^{\circ}$ angle of incidence are depicted in Figure S2 and are labeled A, B, C and D.

A: Electric field is oriented parallel to the long axis of the AuNR and the substrate.

$>$ B: Electric field is oriented perpendicular to the long axis of the AuNR and has projections perpendicular and parallel to the substrate.

$>$ C: Electric field has projections along the long and short axes of the AuNR as well as parallel and perpendicular to the substrate.

D: Electric field is oriented perpendicular to the long axis of the AuNR and parallel to the substrate.

The optical response of the AuNR-AuF system at each of these orientations is analyzed by calculating the far-field transmission spectra and the near-field enhancement at mid gap between the AuNR and AuF illuminating the sample with Gaussian beam. The AuF is modeled as finite disk with diameter larger than $2 \lambda_{0}$ (where $\lambda_{0}$ is the resonance wavelength of the particle-film plasmonic system) and $500 \mathrm{~nm}$ thickness. The AuNR is modeled as cylindrical object capped with hemispheres at the two ends. The bulk dielectric constant values measured by Johnson and Christy ${ }^{1}$ are used as input in simulating the AuF and AuNR. The dielectric data of Palik is used for aluminum oxide. Perfectly matched layer absorbing boundaries are used to define the simulation region. Finer grid size ( $1 \mathrm{~nm}$ and smaller in $\mathrm{x}, \mathrm{y}$ and $\mathrm{z}$ directions) is used around the AuNR and the interface region. The power spectra are recorded in the presence and absence of AuNR on the substrate using a monitor placed $1.86 \mu \mathrm{m}$ above the source, and the extinction spectrum of the AuNR-AuF system is calculated by taking the difference. The spectra obtained following these 
procedures are plotted in Figure S2 on the right side of the corresponding orientations. The corresponding near-field distributions at the mid gap of the AuNR-AuF system are displayed in Figure S3 for each of the orientations.

A
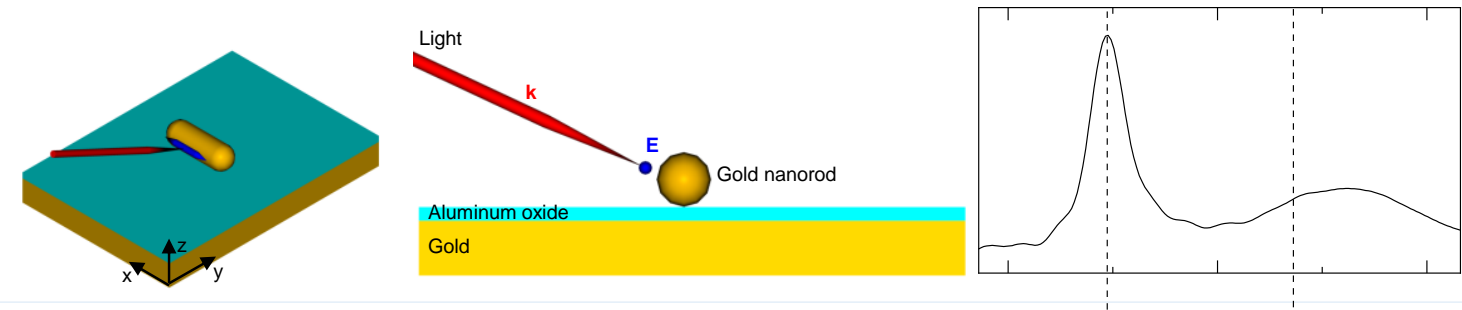

B
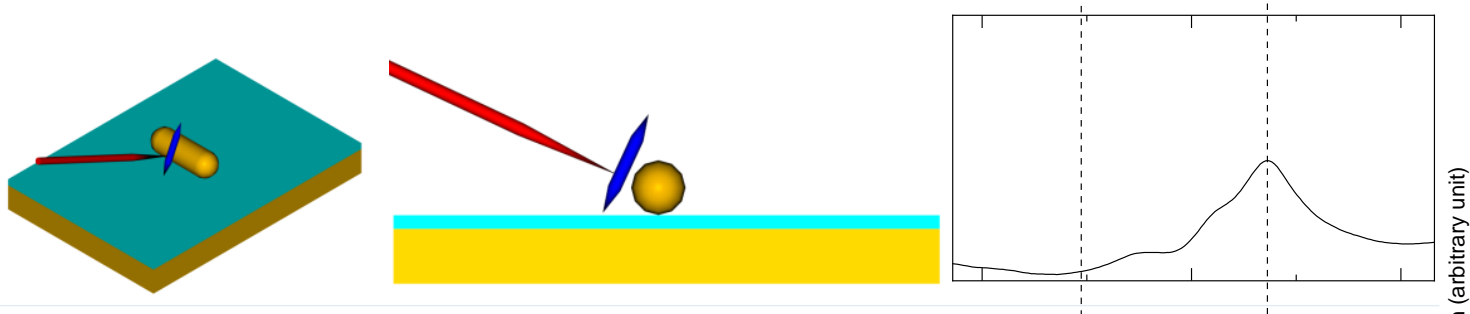

C
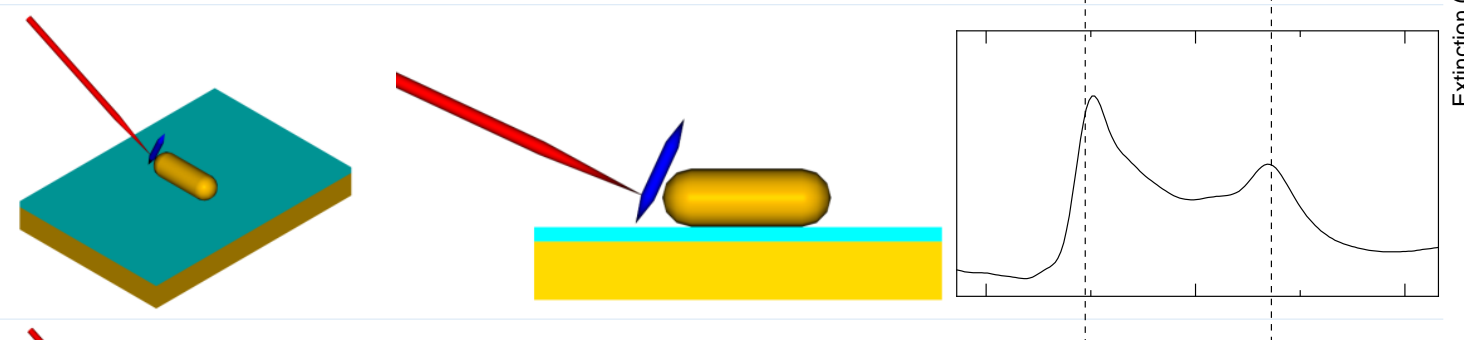

D
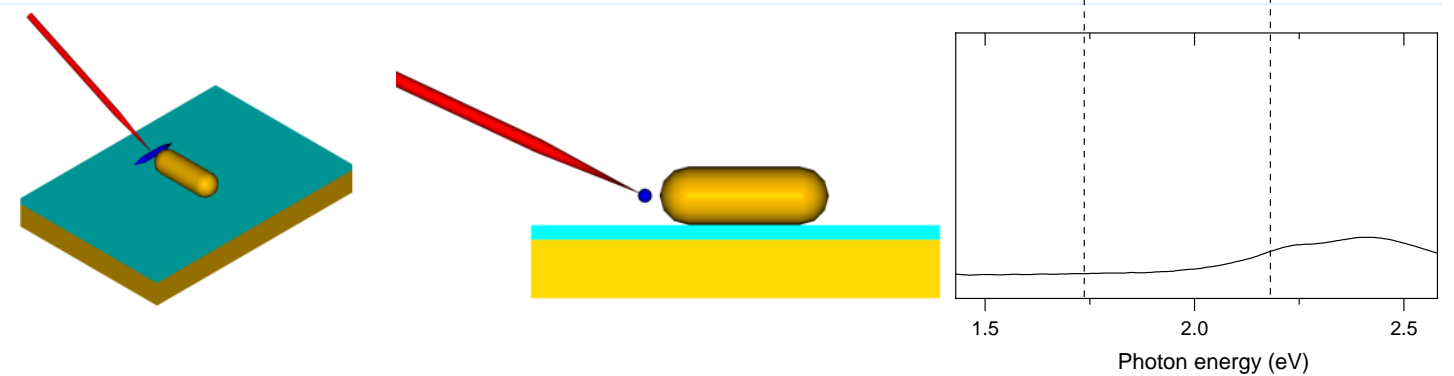

Figure S2 Results of FDTD simulation at four different possible orientation of the electric field (E, indicated by blue arrow) orientation with respect to the AuNR. The two prominent resonances are indicated by the vertical dash lines. In orientation A, $\mathbf{E}$ is parallel to the long axis of the AuNR, a cavity mode that hybridizes with the longitudinal mode of the AuNR is primarily excited. In orientation B, where $\mathbf{E}$ is along the short axis of the AuNR, a cavity mode that hybridizes with the transverse mode of the AuNR is excited. In orientation $\mathbf{C}, \mathbf{E}$ has projections along the long and short axes of the rod, and hence both cavity modes are excited. Orientation D, where $\mathbf{E}$ is parallel to the substrate and along the short axis of the rod, is not effective to excite any of the modes. In all cases, the incidence angle is $45^{\circ}$ with respect to surface normal and the gap between the AuNR and the film is $5 \mathrm{~nm}$. 


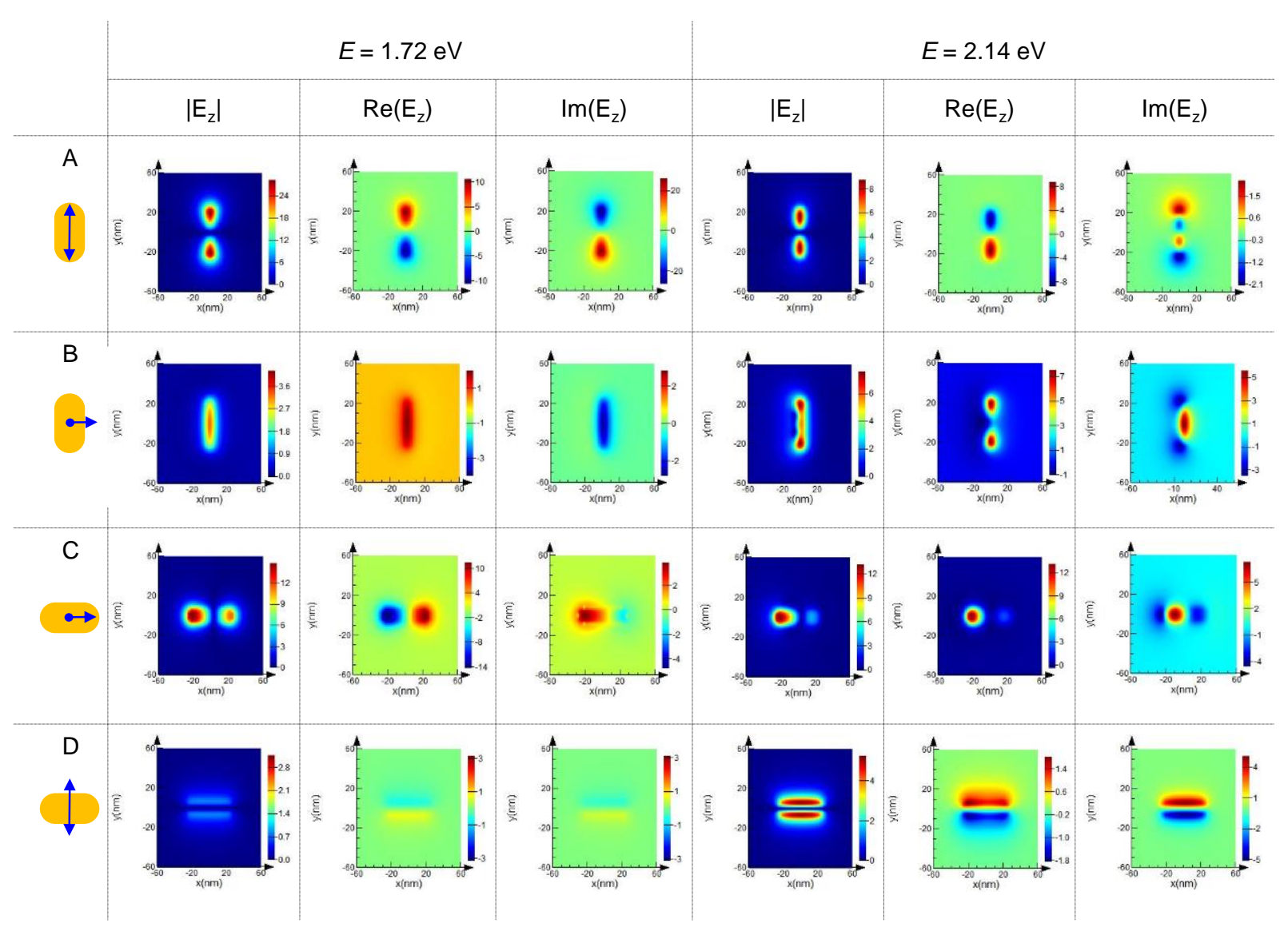

Figure S3 Field distribution and polarization at the mid gap of AuNR-AuF coupled plasmonic system at $1.72 \mathrm{eV}$ and $2.14 \mathrm{eV}$, the spectral positions indicated by the dashed lines in Figure S2. The labels A, B, C and D in column 1 indicate the orientations in Figure S2. For the resonance at $1.72 \mathrm{eV}$, which is due to the hybridization of the cavity mode with the fundamental longitudinal plamon mode of the AuNR, maximum field enhancement is obtained when the excitation field is oriented along the long axis of the rod and parallel to field (orientation A). On the other hand, for the resonance around $2.14 \mathrm{eV}$, maximum field enhancement is obtained when the electric field has projection perpendicular to the film. For excitation field oriented parallel to the substrate and perpendicular to the long axis of the rod, no field enhancement is observed in the gap region, indicating the absence of AuNR-AuF plasmon coupling as shown for orientation D.

Next, we repeat the calculations for normal incidence considering electric field orientation parallel and perpendicular to the long axis of the AuNR as shown in Figure S4a. These orientations are used to compare the results of the FDTD simulation using a Gaussian source and a totalfield/scattered-field (TFSF) source. Comparing the results in Figures S4b and S4c, it can be seen that the results are essentially the same. In addition, the results obtained at normal incidence reproduces our experimental observation satisfactorily. Therefore, the TFSF source is used in our further calculation because of its simplicity to obtain background free scattering cross-section. In all the simulations from now on, the TFSF source is introduced in the region where the grid size is $0.5 \mathrm{~nm}$ in $\mathrm{x}, \mathrm{y}$ and $\mathrm{z}$ directions. Except for the results in Figures S2-S4, the dielectric spacing has $1 \mathrm{~nm}$ overlap with the AuNR side facing the substrate, and a mesh override is applied such that the overlapped region becomes dielectric. This creates a facet width of $12.5 \mathrm{~nm}$ at the bottom 
of the AuNR surface that interacts with the dielectric spacer. We note that increasing the facet width shifts the resonance wavelength to the red.

(a)
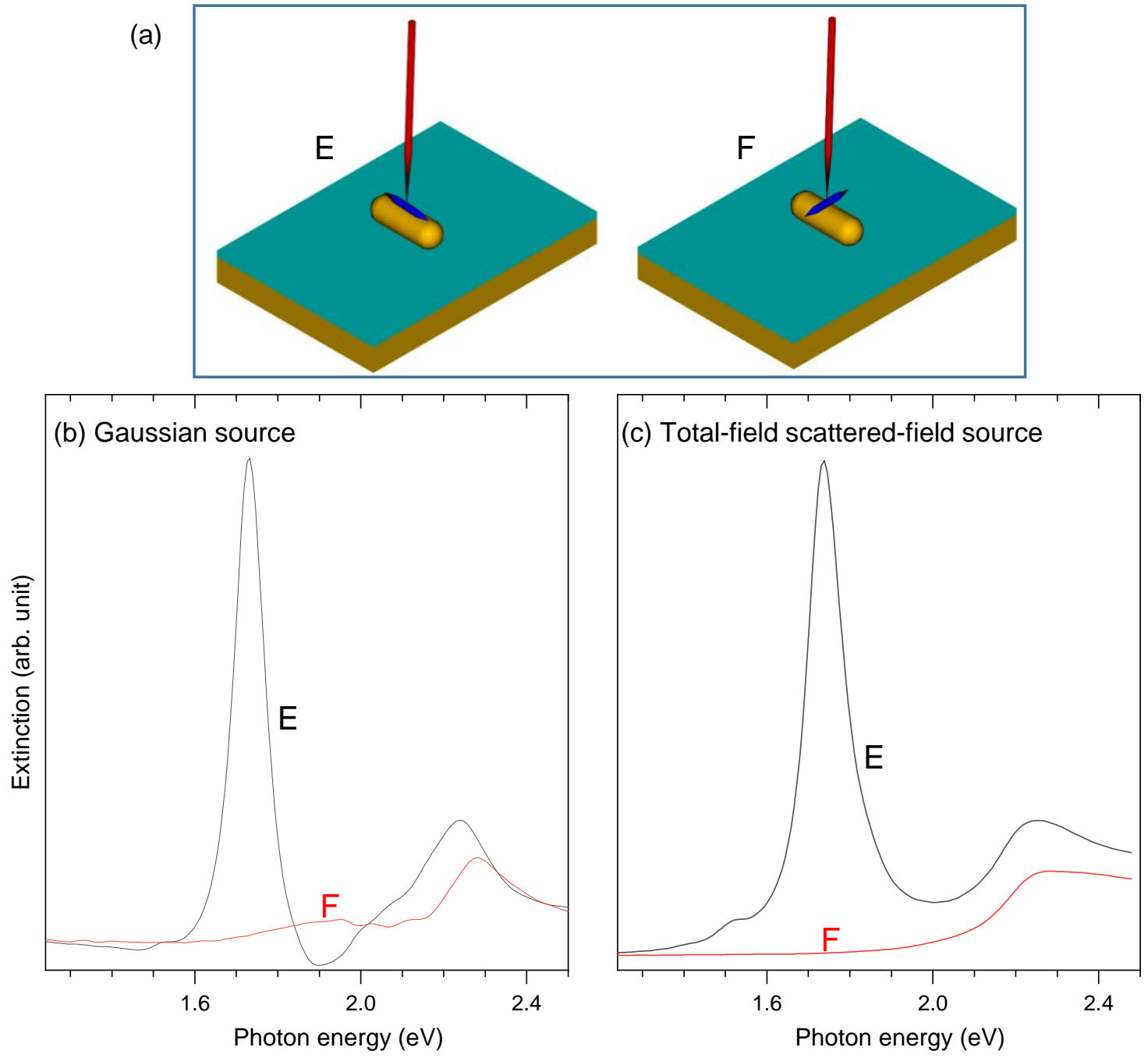

Figure S4 (a) Schematic showing normal incidence with electric field orientation parallel to the long axis of the rod (orientation E) and perpendicular to the long axis of the rod (orientation F). (b, c) Comparison of the results for Gaussian source (b) and total-field scattered field sources (c). The aluminum oxide spacer thickness is $5 \mathrm{~nm}$. The results for the two sources are essentially the same. The dip at $\sim 1.9 \mathrm{eV}$ in (b) for orientation E results from background subtraction, which suggests that the optical response of the gold film can be influenced slightly by the presence of the gold nanorod. The small peak at $1.52 \mathrm{eV}$ in (c) for orientation E disappears when the grid size around the AuNR-AuF interface is reduced to $0.5 \mathrm{~nm}$, indicating that it originates from simulation artefact. 

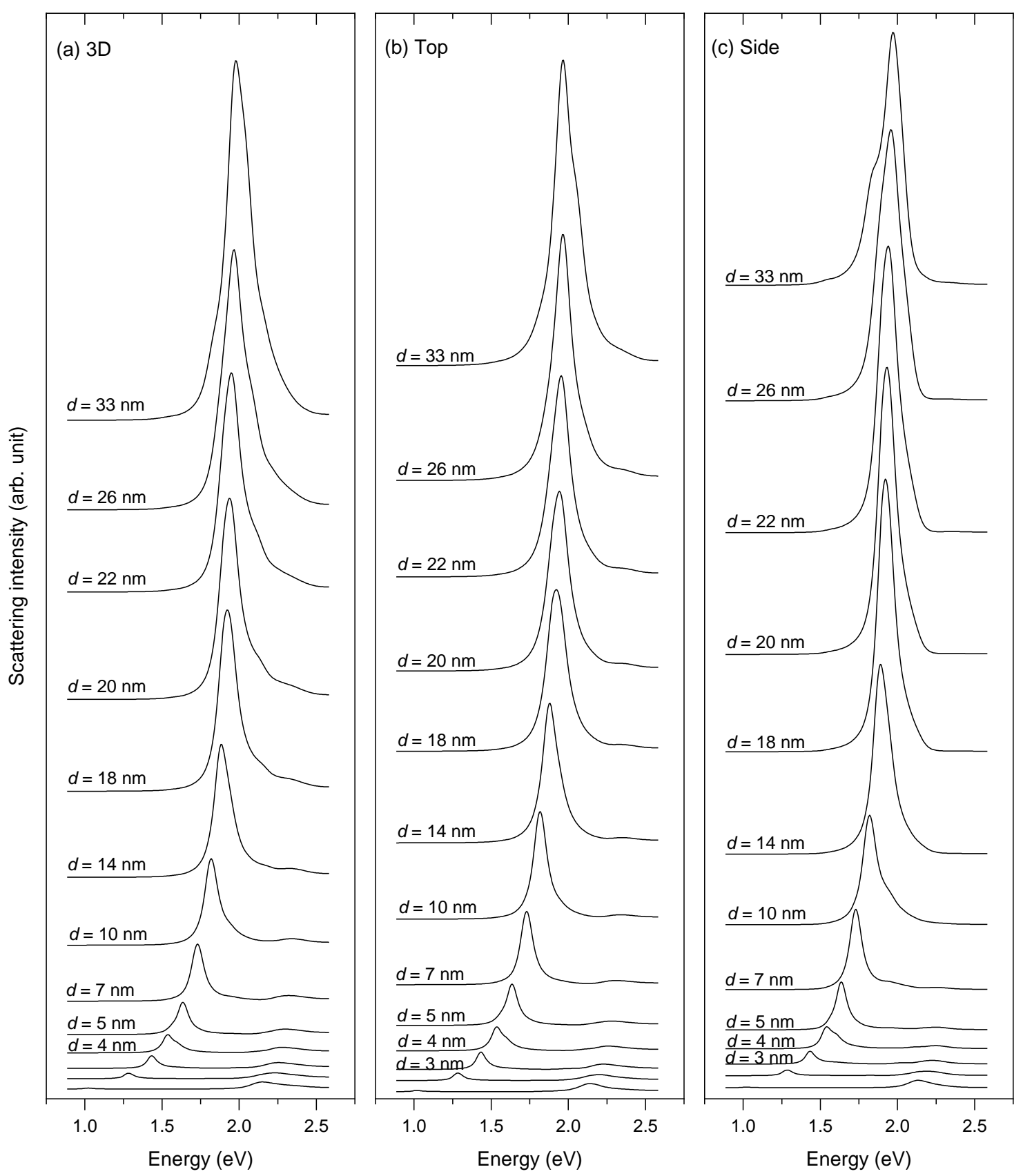

Figure S5 Dependence of peak cross-section on collection angle. (a) When the light scattered at all angles is collected, the scattering cross-section increases continuously with increasing AuNR-AuF separation $d$. (b) When the scattered light is monitored above the sample on a plane parallel to the gold film, the scattering cross-section increases continuously as in (a). (c) When the scattered light is monitored on planes perpendicular to the gold film, the peak value of the scattering cross-section increases up to $d=20 \mathrm{~nm}$, and then decreases. The trend in (c) agrees with the experimental observation in Figure 2a. Possible explanation for this trend is given in Figure S6. 


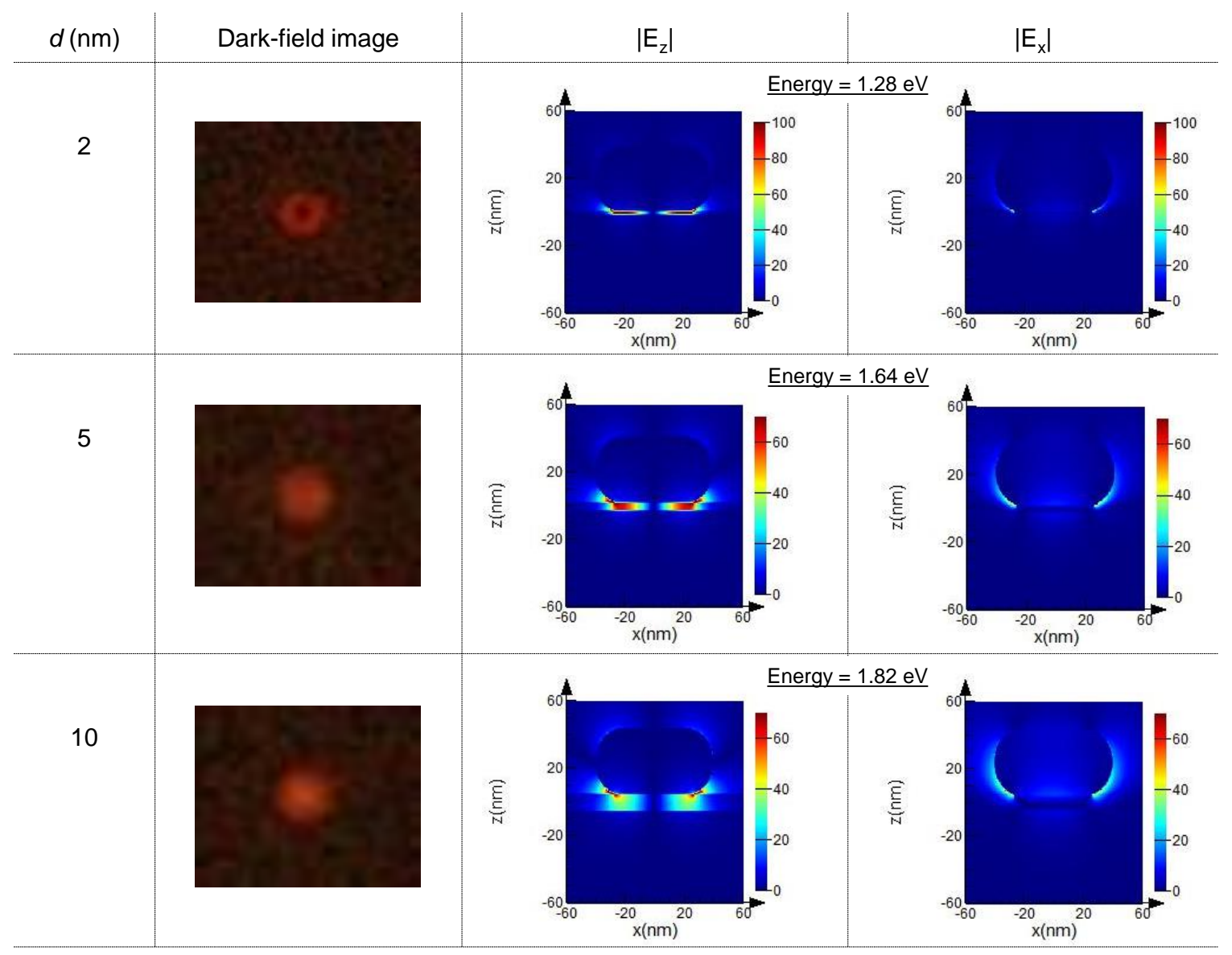

Figure S6 The trend in Figure S5 can be explained in terms of contribution of the scattering by the transverse cavity mode (electric field confined in the gap and oriented perpendicular to the film along the z-direction, see third column) and antenna mode (electric field is distributed around the ends of the AuNR and is oriented parallel to the sample plane along the $\mathrm{x}$-direction (fourth column). We note that although the scattering spectra for different gap thicknesses have been measured on different dates that can result in slightly different alignment condictions, in general, the relative scattering intensity appears to maximize in the $d=9-20 \mathrm{~nm}$ range. On the other hand, the calculated scattering cross-section increases continuously with increasing $d$ if the light scattered at all angles is collected as shown in Figure S5a. Similar gap dependence of scattering intensity (Figure S5b) is obtained when the scattering is monitored on a plane parallel to the gold film. This discrepancy between experimental observation and theoretical prediction can be attributed to different contribution of the cavity and antenna modes. For the cavity mode, the near-field vector is perpendicular to the plane and scatters radially, resulting in donut shaped dark-field images as shown for $d=2 \mathrm{~nm}$. For the antenna mode, the near-field vector is oriented parallel to the plane and radiates preferentially perpendicular to the plane. The scattering from the antenna mode becomes dominant as the gap length increases resulting in the dark-field images that have isotropic solid structures as shown for $d=$ 5 and $10 \mathrm{~nm}$. The continuous increase of the calculated cross-section is due to the more effective coupling of the antenna mode to the far-field than the cavity mode that shows more pronounced near-field enhancement. ${ }^{2}$ The relative scattering cross-section (Figure $2 \mathrm{c}$ and S5c) monitored on a plane perpendicular to the sample plane that detects radially scattered photons shows reasonable agreement with the intensity trend observed in the experiment. This agreement may indicate that our optical setup is biased toward detecting scattering from the cavity modes while the theory exaggerates the efficiency of scattering by the antenna modes. 


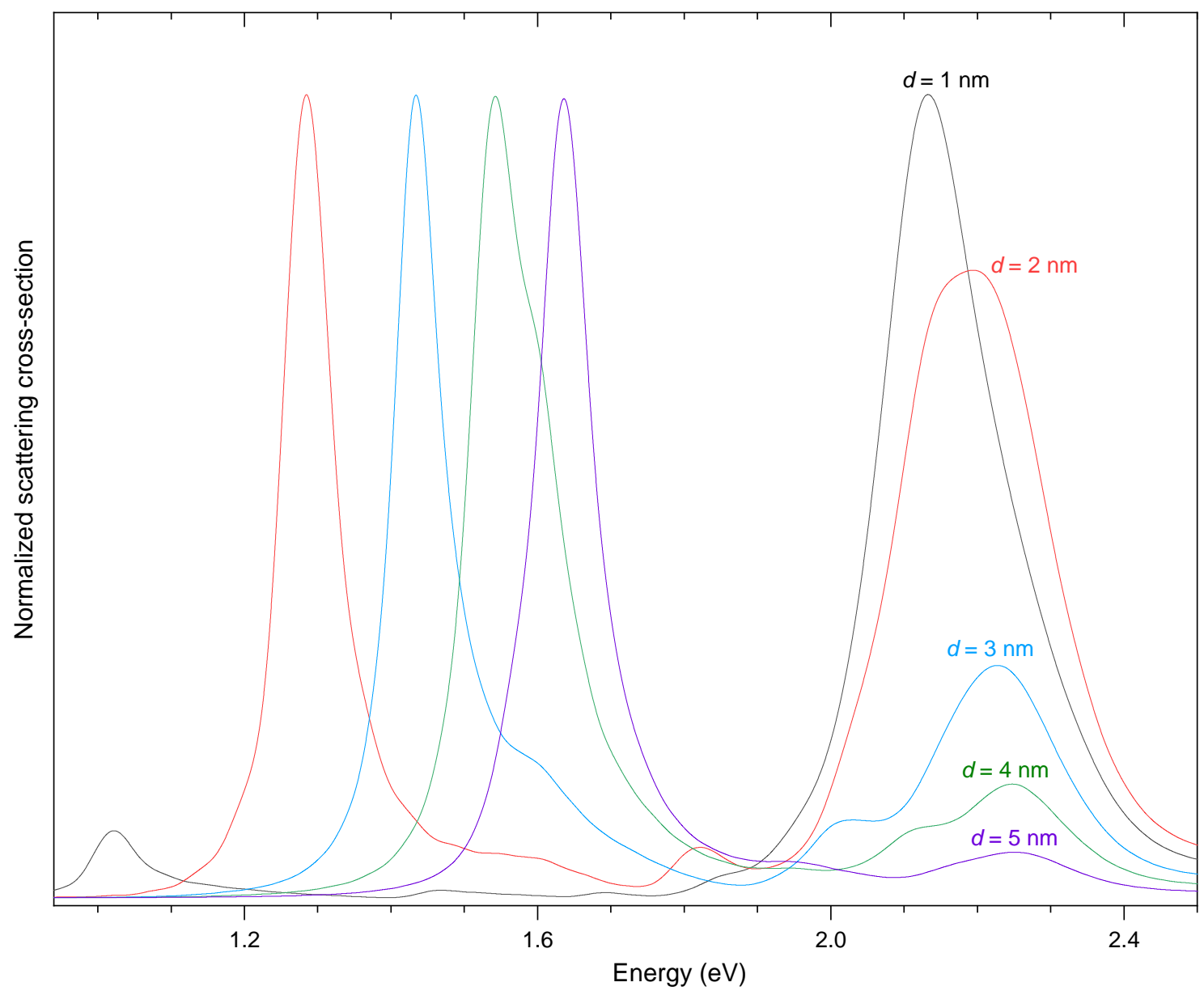

Figure S7 Normalized scattering cross-sections showing the relative intensities of the low and high energy peaks as the gap size decreases from $5 \mathrm{~nm}$ to $1 \mathrm{~nm}$. 


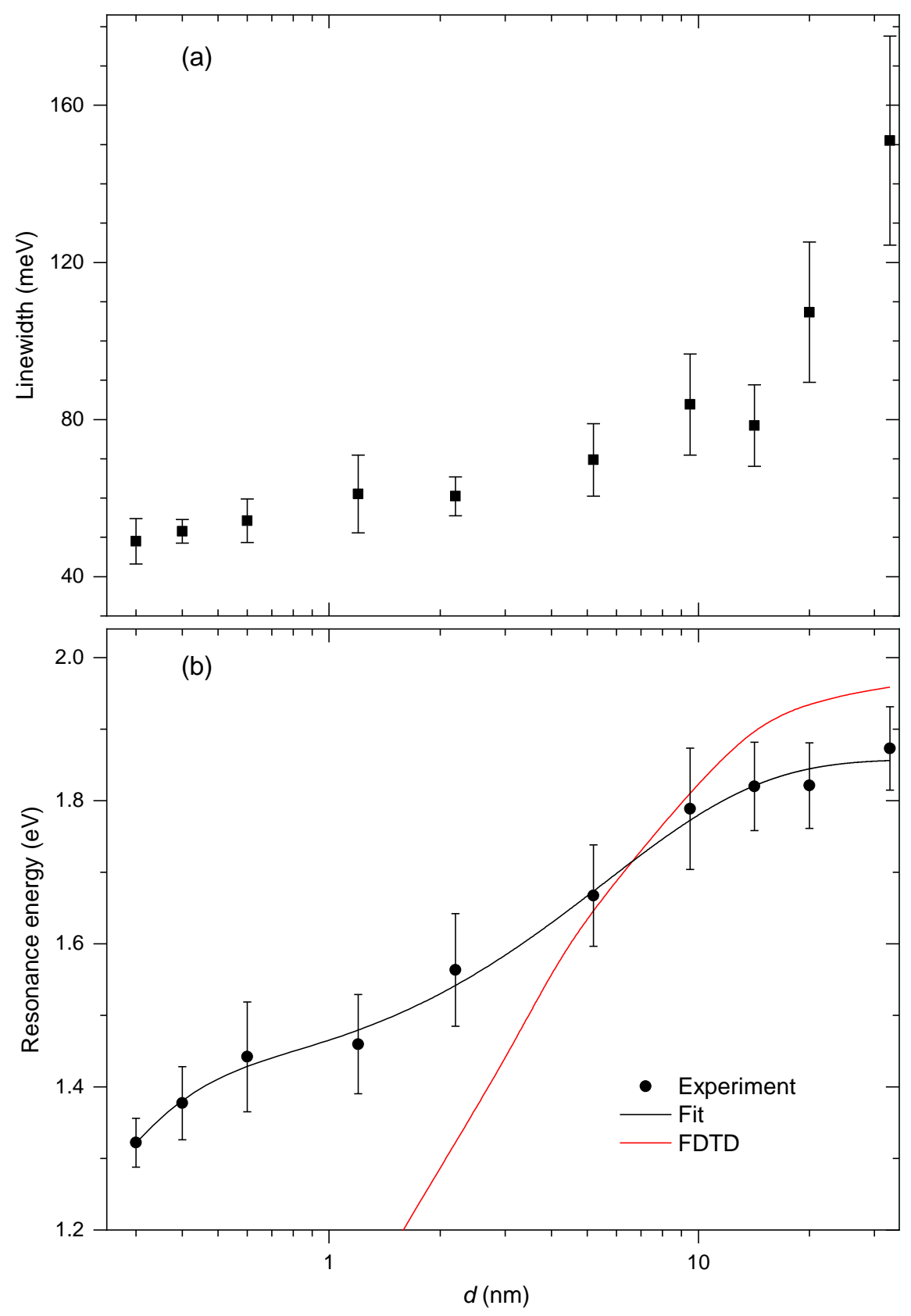

Figure S8 Same as Figure $3 \mathrm{~b}$ and $3 \mathrm{c}$ plotted here using log scale for the gap length $d$. 


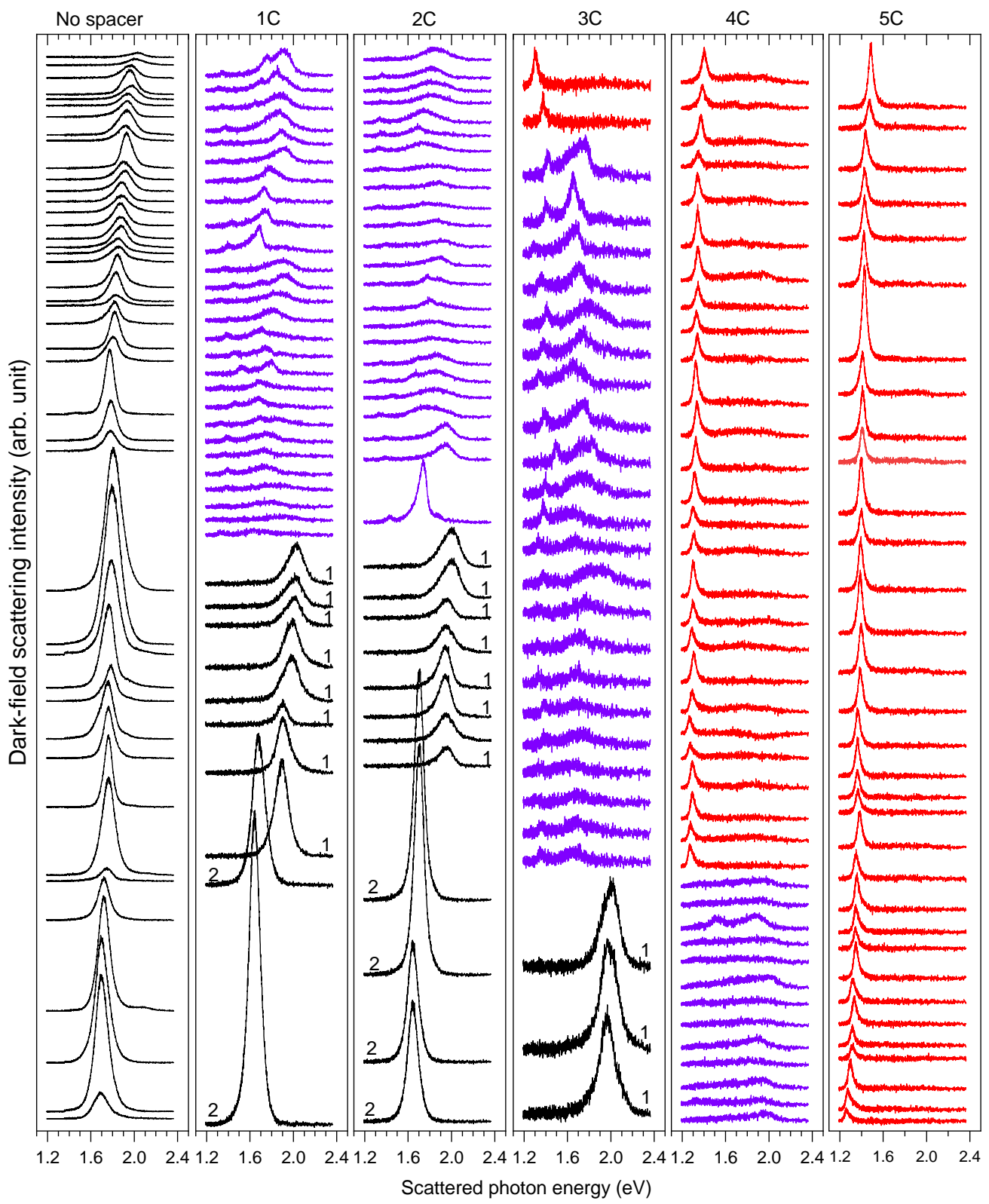

Figure S9 The evolution of AuNR-AuF gap plasmon resonances as the aluminum oxide growth cycles is reduced from 5 ALD cycles $(5 \mathrm{C})$ to zero. At $5 \mathrm{C}$, the scattering spectra are primarily characterized by a dominant single peak in the near-infrared region with weak signature of broad resonances on the shorter wavelength side. At 4C, the broad resonances become apparent in all the scattering spectra recorded, and the sharp peaks in the near-infrared region do not appear in some of the spectra. At 3C, the sharp peaks in the near-infrared region are rarely observed, while multipeak spectra with weak scattering intensity (violet lines) are observed for the majority of the AuNRs. In addition, spectra with single peak and stronger scattering intensity (black lines) are observed for some of the AuNRs. The single peak spectra become more frequent as the ALD cycles are reduced to $2 \mathrm{C}$ and $1 \mathrm{C}$, and become the only spectra for AuNRs directly deposited on the AuF. For 3C-1C, the black spectra are labeled 1 and 2 based on their peak positions. 

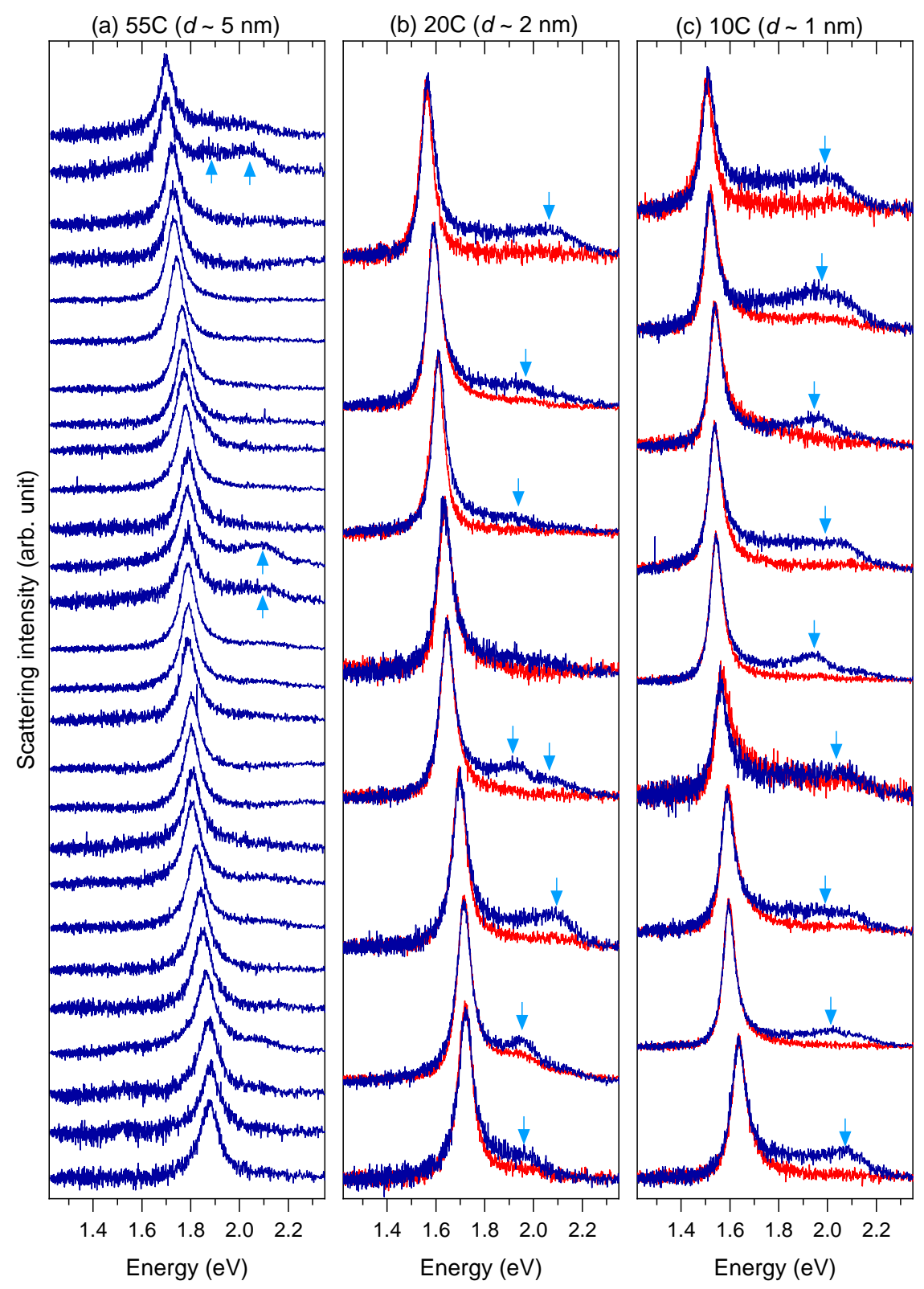

Figure S10 Experimental demonstration that detection of high order modes can depend on experimental settings. The experimental results presented in Figures 2a-b, 3, 4 and S9 are obtained using the center one pixel of the CCD camera of our spectrometer in order to minimize inhomogeneous linewidth broadening and to improve the spatial resolution in obtaining single particle spectra. In this example, the effect of CCD camera pixel binning is demonstrated by comparing the spectra obtained using the center 10 pixels (blue lines) and center one pixel (red lines). The blue arrows indicate peaks due to high order cavity modes. (a) For relatively large spacing $(d \approx 5 \mathrm{~nm})$, for only 3 out of 27 spectra of individual AuNRs coupled to AuF show peaks of high order modes. For $d \approx 2 \mathrm{~nm}(\mathrm{~b})$ and $d \approx 1 \mathrm{~nm}(\mathrm{c})$, peaks due to high order modes are observed for almost all AuNRs when the center 10 pixels are used but the peaks are lost in the background when only the center one pixel is used. 


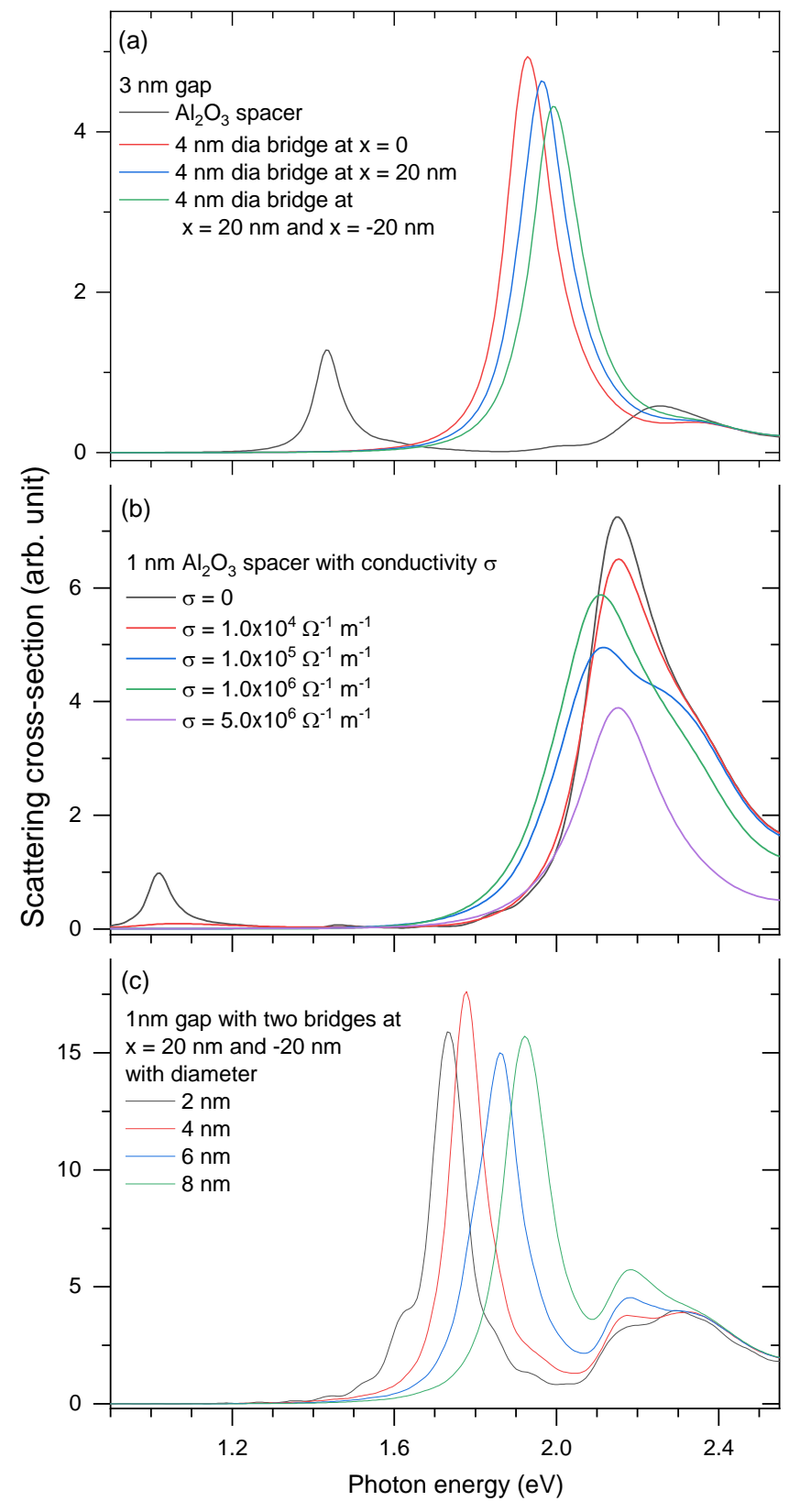

Figure S11 (a) Effect of cylindrical gold bridge position. The diameter (dia) of the bridge is specified on the plot. (b) and (c) Same as Figure 5 for spacer size of $1 \mathrm{~nm}$. 


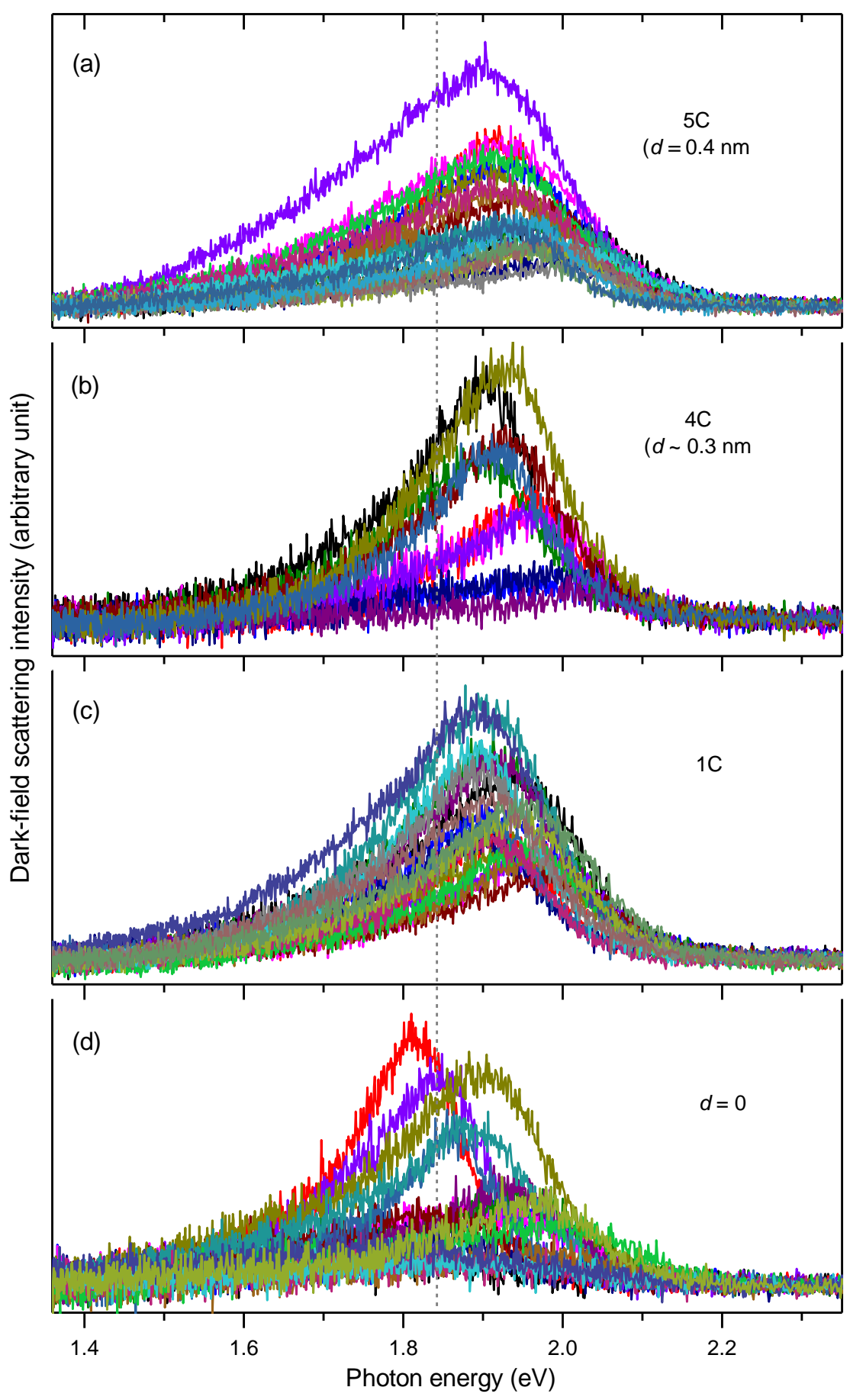

Figure S12 Scattering properties of gold nanospheres on AuF for aluminum oxide spacer layer thickness obtained in the 5C - 0 ALD cycles. The overall spectral properties remain essentially the same when the thickness of the spacer layer is decreased to direct contact, with the exception of small red-shifting toward the conductive limit that matches the resonances of the AuNRs directly placed on AuF. The vertical dashed line at $1.84 \mathrm{eV}$ is the average energy obtained for AuNRs on AuF (see text in the main manuscript), and is included here for comparison. 


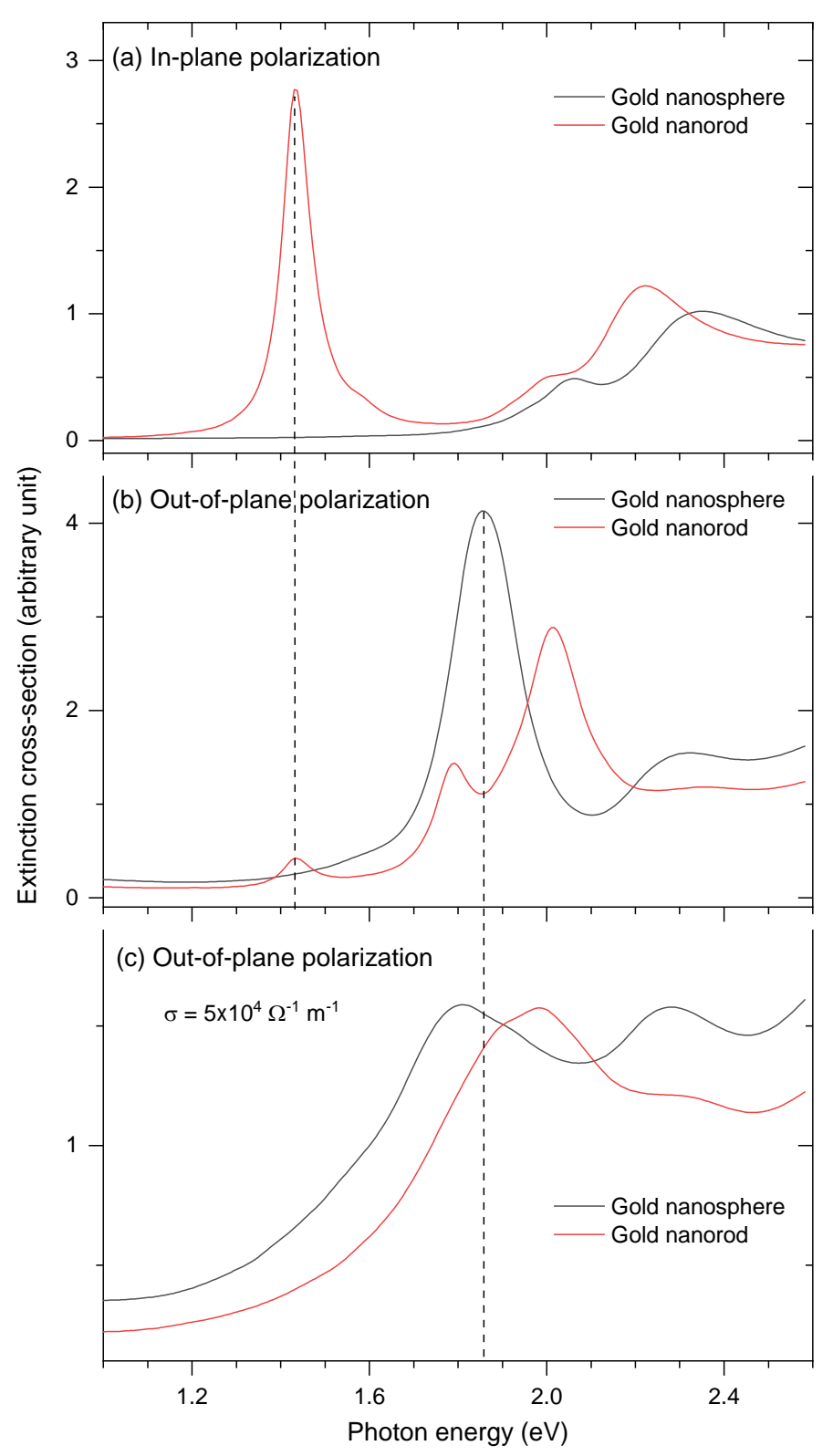

Figure S13 Comparison of optical responses of gold nanorod (width $40 \mathrm{~nm}$ and length $80 \mathrm{~nm}$ ) and gold nanosphere (diameter $56 \mathrm{~nm}$ ) coupled to gold film through $3 \mathrm{~nm}$ spacing. The nanorod and nanosphere have approximately the same volume. (a) The electric field of the excitation source is oriented parallel to the film and along the long axis of the rod. Clearly, the cavity mode resonance that peaks at $1.43 \mathrm{eV}$ for the gold nanorod coupled to the gold film does not exist in the nanosphere-film system. (b) The excitation field is oriented perpendicular to the film. For gold nanosphere, the strong peak at $1.85 \mathrm{eV}$ is due to dipole and image dipole particle-film coupling, which is less favorable for the anisotropic gold nanorod. For this outof-plane polarization, the strong cavity resonance of the gold nanorod is diminished drastically as can be seen comparing the peak values at $1.43 \mathrm{eV}$ in (a) and (b). (c) Introduction of conductivity to the dielectric spacer kills the sharp resonances of the gold nanorod. For the gold nanosphere, the effect of the conductivity is limited to broadening, while the shift in the peak positions is minor. 
1. Johnson, P. B.; Christy, R. W., Optical-constants of noble-metals. Phys. Rev. B 1972, 6 (12), 4370-4379.

2. Bozhevolnyi, S. I.; Sondergaard, T., General properties of slow-plasmon resonant nanostructures: nano-antennas and resonators. Opt. Express 2007, 15 (17), 10869-10877. 